\title{
The Effect of Retraining on Treatment Success, Quality of Life, and Metabolic Parameters in Patients with Type 1 Diabetes Using an Insulin Pump
}

\author{
Basak Ozgen Saydam Fatma Yilmazmis Nalan Aydin Belgin Bektas \\ Simge Yilmaz Umit Cavdar Secil Ozisik Baris Akinci \\ Division of Endocrinology and Metabolism, Dokuz Eylul University, Izmir, Turkey
}

\section{Significance of the Study}

- In this study, a short-term retraining program was an efficient method to improve the level of knowledge and practice skills of patients with type 1 diabetes using continuous subcutaneous insulin infusion. Patients with poor metabolic control were the best candidates for such a program because retraining significantly improved glycemic control in this subgroup of patients.

\section{Keywords}

Glycemic control · Insulin pump · Quality of life · Type 1 diabetes · User training

\begin{abstract}
Objective: To investigate the effect of insulin pump user retraining on treatment success, quality of life, and metabolic parameters of patients with type 1 diabetes using continuous subcutaneous insulin infusion. Subjects and Methods: $A$ total of 35 subjects participated in this prospective study. All patients were given insulin pump user retraining. Their knowledge level and application skills, metabolic parameters, quality of life, and satisfaction from treatment were evaluated at baseline and after 6 months. Results: There was significant improvement in patients' knowledge and application skills after insulin pump user retraining (self-assess-
\end{abstract}

\begin{tabular}{ll}
\hline KARGER & (c) 2017 S. Karger AG, Basel \\
$\begin{array}{l}\text { E-Mail karger@karger.com } \\
\text { www.karger.com/mpp }\end{array}$ & $\begin{array}{l}\text { This is an Open Access article licensed under the terms of the } \\
\text { Creative Commons Attribution-NonCommercial 3.0 Un- } \\
\text { ported license (CC BY-NC) (www.karger.com/OA-license), } \\
\text { applicable to the online version of the article only. Distribu- } \\
\text { tion permitted for non-commercial purposes only. }\end{array}$
\end{tabular}

ment of user skills: $69.7 \pm 11.5$ vs. $76.3 \pm 11.3, p<0.001$; knowledge level on technical issues: $3.3 \pm 1.1$ vs. $4.1 \pm 1.8$, $p=0.003$; glucose monitoring: $27.1 \pm 5.8$ vs. $29.2 \pm 5.6, p=$ 0.006 ; management of hyperglycemia: $13.1 \pm 3.2$ vs. $15.7 \pm$ $3.4, p<0.001$; management of pump and infusion site problems: $8.8 \pm 2.6$ vs. $10.6 \pm 2.6, p=0.001)$. Hemoglobin $(\mathrm{Hb}) \mathrm{A}_{1 \mathrm{c}}$ levels of patients with poor glycemic control improved after retraining ( $8.61 \% \pm 0.78$ vs. $8.23 \% \pm 0.79, p=0.02)$. However, no significant improvement in quality of life and treatment satisfaction parameters were found. Conclusion: Management of type 1 diabetes in insulin pump users can be significantly improved by retraining. Even a basic short-term retraining program helps patients to increase their knowledge level and ability to more effectively use the insulin pump. The fact that retraining significantly improves glycemic parameters in patients with poor metabolic control indicates that priority should be given to this group of patients.

Basak Ozgen Saydam, MD

Division of Endocrinology

Dokuz Eylul University, Mithatpasa Street 1606

TR-35340 Izmir (Turkey)

E-Mail basakozgen@gmail.com 
Further studies with individualized training programs in larger sample sizes with long-term follow-up are needed to establish the importance of retraining and create re-education plans for patients with type 1 diabetes using an insulin pump.

(c) 2017 S. Karger AG, Basel

\section{Introduction}

Poorly controlled type 1 diabetes is associated with long-term microvascular and macrovascular complications [1]. Intensification of insulin treatment has been shown to be effective in preventing chronic complications of diabetes [1-3]. Continuous subcutaneous insulin infusion (CSII), a method of insulin delivery, mimics the physiological insulin secretion pattern most successfully and allows patients to adjust their basal and mealtime bolus insulin doses [4]. Treatment of patients with type 1 diabetes with CSII offers better flexibility for patients and enables them to meet their personalized needs in management of diabetes [4-6]. When used properly in appropriate patients, CSII has been shown to be more efficient than multiple daily injections in keeping blood glucose levels at target levels without getting exposed to an increased risk of hypoglycemia [7, 8]. Patients using an insulin pump have also shown better quality of life (QoL) scores when compared to their counterparts taking multiple daily injections $[6,9]$. Due to technological developments, insulin pumps have become easier to use over time. However, the success of CSII still depends on an efficient patient diabetes education and pump training plan $[6,10,11]$. Intensive insulin pump training consists of 2 major issues: diabetes self-management using insulin pump therapy and technical training on the insulin pump [12]. Self-management of advanced insulin therapy includes carbohydrate counting, infusion site management, and prevention and management of hypoglycemia and diabetic ketoacidosis [13]. After acquiring sufficient self-management skills, the second part of education consists of technical training $[12,13]$. There are many methods for education, which include group education, personalized education, and newly emerging remote technology communication [14]. After a successful initial education, periodic re-evaluation visits and established retraining protocols are essential [15-17]. Parental knowledge has been shown to be associated with better glycemic control in adolescents and children [11]. Transition to adulthood and taking responsibility of their own disease increase the need of re-education in type 1 diabetic patients diagnosed in childhood. Re-education is also thought to be important in preventing diabetic com- plications [17]. Jenkins et al. [17] showed that despite a detailed education at pump initiation, some patients did not have appropriate knowledge and were not able to manage insulin pump failure and decrease the risk of hyperglycemia and diabetic ketoacidosis, revealing the importance of retraining. Hence, in this study we aimed to investigate the effects of insulin pump user retraining on treatment success, QoL, and metabolic parameters of patients with type 1 diabetes using CSII.

\section{Materials and Methods}

Thirty-seven patients were included in the study; however, 2 subjects were excluded as they did not attend posttraining visits. All participants were followed up by endocrinologists at Dokuz Eylul University Hospital. Written informed consent was obtained from all the subjects. The study was approved by the Ethics Committee of Dokuz Eylul University (08.01.2015,1864-GOA, 2015 01-12).

\section{Study Design}

Patients were evaluated at baseline and 6 months after insulin pump user retraining was completed (Fig. 1). Patients completed a series of education meetings to improve their practices concerning CSII management and carbohydrate counting. Education groups consisted of 8-10 participants. Qualified dieticians and diabetes education nurses gave the education courses. PowerPoint presentations were made under supervision of a staff clinical endocrinologist. The retraining program consisted of 4 parts (Fig. 2). In the first part, patients' general knowledge about the disease that included monitoring and management of blood glucose levels, awareness of hypo/hyperglycemia, record keeping, and glucagon administration, was assessed. In the second part, the patients were given an education course. The course mainly consisted of information on carbohydrate counting, infusion site management, hypoglycemia prevention, management and prevention of diabetic ketoacidosis, and site preparation and care to prevent infection. In the third part of the retraining program, technical knowledge and skills were emphasized including practical tips on pump usage, glucose monitoring, adjusting basal doses, and entering and managing bolus doses. The last part of the presentation was comprised of management of certain circumstances such as acute illnesses, exercise, emergency situations holidays, and pump site infections. The retraining program was a 1-day course that also included a question and answer session.

The authors generated a questionnaire to assess patients' education level, knowledge, and application skills. The questionnaire had been previously validated in CSII users (unpublished data). The questionnaire was filled in at baseline and also 6 months after the retraining program. Briefly, the questionnaire was divided into 3 parts: patients' characteristics $=$ information on demographic properties of patients such as age, sex, body mass index (BMI), education level, marital status, type of social security, occupation, social life and smoking habits were obtained; diabetes and its complications $=$ the characteristics of diabetes such as the duration of the disease and chronic complications related to diabetes were assessed; and insulin pump user skills = questions regarding insulin pump usage were asked to rank carbohydrate counting skills and technical knowledge on the device. Practical application skills were 


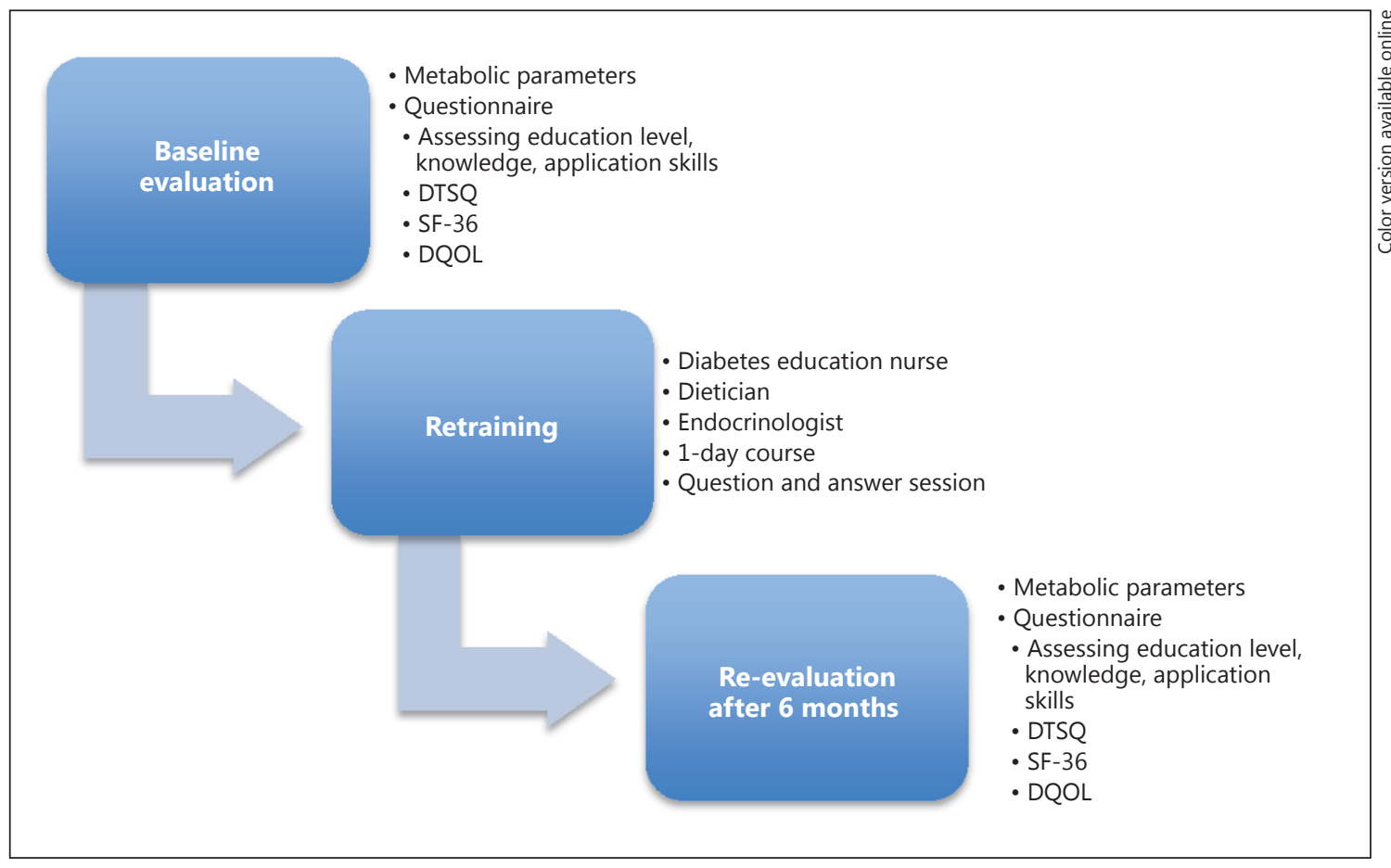

Fig. 1. Study design. DTSQ, Diabetes Treatment Satisfaction Questionnaire; SF-36, Medical Outcome Study Health Survey 36-Item Short Form; DQOL, Diabetes Quality of Life.

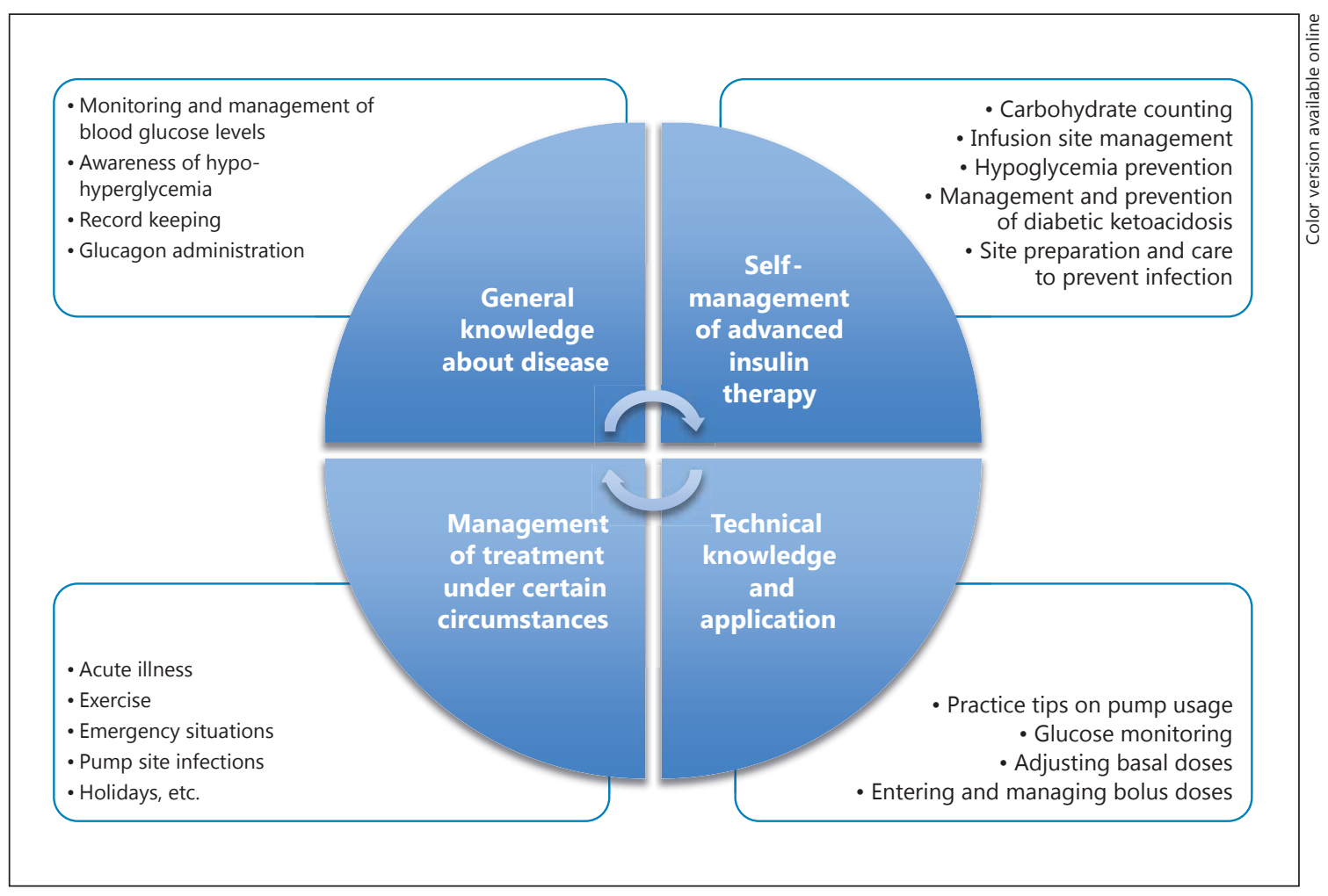

Fig. 2. The content of the retraining program. 
Table 1. Comparison of patients' knowledge levels and application skills before and 6 months after user retraining

\begin{tabular}{|c|c|c|c|}
\hline & $\begin{array}{l}\text { Before training } \\
(n=35)\end{array}$ & $\begin{array}{l}\text { After training } \\
(n=35)\end{array}$ & $p$ \\
\hline Self-assessment of CSII user skills & $69.7 \pm 11.5$ & $76.3 \pm 11.3$ & $<0.001$ \\
\hline Knowledge level on technical issues & $3.3 \pm 1.1$ & $4.1 \pm 1.8$ & 0.003 \\
\hline Glucose monitoring & $27.1 \pm 5.8$ & $29.2 \pm 5.6$ & 0.006 \\
\hline Management of hypoglycemia & $10.7 \pm 1.8$ & $11.3 \pm 1.8$ & 0.166 \\
\hline Management of hyperglycemia & $13.1 \pm 3.2$ & $15.7 \pm 3.4$ & $<0.001$ \\
\hline Approach to pump, infusion set, and infusion site problems & $8.8 \pm 2.6$ & $10.6 \pm 2.6$ & 0.001 \\
\hline Pump management under specific circumstances such as illness and exercise & $2.5 \pm 1.1$ & $2.7 \pm 1.4$ & 0.468 \\
\hline
\end{tabular}

Data are shown as means \pm SD. CSII, continuous subcutaneous insulin infusion.

assessed in detail. This part included questions about insulin pump functions, insulin pump warning signals, ability to set up and change basal doses, temporary basal doses, and manual bolus doses. Also the frequencies of hypo- and hyperglycemia and how the patients handled these situations, their level of knowledge on diabetes, strategy to solve problems such as pump, infusion set and infusion site problems, acute illness, and exercise were assessed. A certified diabetes educator interpreted the questionnaire.

Metabolic parameters were studied at baseline and repeated 6 months after the retraining was completed. Blood was taken from the cannulated antecubital vein between 8:00 a.m. and 9:00 a.m. after 8-h overnight fasting, and blood samples were transferred into tubes containing EDTA and $\mathrm{NaF}$, as well as tubes suitable for serum preparation. Glucose, $\mathrm{HbA}_{1 \mathrm{c}}$, lipids, alanine aminotransferase, and microalbuminuria ( $\mathrm{mg} / \mathrm{g}$ to creatinine) levels were measured by standardized methods with appropriate quality control and quality assurance procedures. QoL was assessed using the Medical Outcome Study Health Survey 36-Item Short Form (SF36) and Diabetes Quality of Life (DQOL). The Diabetes Treatment Satisfaction Questionnaire (DTSQ) was used to determine treatment satisfaction. These surveys were filled at baseline as well as 6 months after retraining.

\section{Statistical Analysis}

Statistical analysis was performed with IBM SPSS Statistics for Mac Version 20 (released 2011, IBM Corp., Armonk, NY, USA). Numeric variables were summarized as mean values and standard deviation. Categorical variables were shown numerically with percentages in parenthesis. A paired $t$ test was used for comparison of baseline and postintervention variables. Categorical variables were compared using the $\chi^{2}$ test. A $p$ value $<0.05$ was considered as statistically significant.

\section{Results}

The mean age was $34.9 \pm 11.3$ years. There were $26 \mathrm{fe}$ males $(74.3 \%)$ and 9 males $(25.7 \%)$. The mean calculated BMI was $24.1 \pm 3.4$. Most patients were highly educated: $23(65.7 \%)$ had graduated from university, $8(22.8 \%)$ from high school, 2 (5.7\%) from middle school, 1 (2.9\%) from primary school, and 1 (2.9\%) was nonliterate. Twenty patients $(57.1 \%)$ were married, 12 patients $(34.3 \%)$ were single, and 3 patients (8.6\%) were divorced. Four patients (11.4\%) were students, 4 (11.4\%) retired, 6 (17.1\%) laborers, 11 (31.4\%) public servants, 6 (17.1\%) self-employed, and 4 (11.4\%) were not working at the time of the study.

The mean duration of diabetes was $197 \pm 113$ months (range: 18-432). Subjects were on CSII for a mean period of $72 \pm 48$ months (range: $5-156$ ). Seven (20\%) patients were smokers. Microvascular complications were present in $8(22 \%)$ patients, while none of the subjects had any macrovascular complications of diabetes. None of the patients had experienced diabetic ketoacidosis during the time period they were on CSII.

Insulin pump user retraining was associated with significant improvements in patients' knowledge levels and application skills (Table 1). No statistically significant improvement was observed in metabolic parameters after user retraining. On the other hand, $\mathrm{HbA}_{1 \mathrm{c}}$ levels dropped significantly after retraining $(8.61 \pm 0.78 \%$ vs. $8.23 \pm$ $0.79 \%, p=0.02$ ) in a subgroup of patients with poor metabolic control, which was defined as having a basal $\mathrm{HbA}_{1 \mathrm{c}}$ level more than $7.5 \%$. Although there was a decrease in hypoglycemic episodes (defined as having blood glucose levels $<70 \mathrm{mg}$ /dL by self-glucose measurements) after retraining, the difference was not statistically significant ( $2.37 \pm 0.12$ vs. $2.23 \pm 0.12$ times/week, $p=0.201$, in the whole study group; $2.43 \pm 0.17$ vs. $2.26 \pm 0.18$ times/week, $p=0.257$, in patients with poor metabolic control). Although user retraining provided some improvements in QoL measured by the SF-36, DQOL, and DTSQ questionnaires, the differences were not significant in either the whole group or among those with poor basal metabolic 
control. There were no statistically significant differences before and after retraining in terms of SF-36 parameters such as physical function, physical role difficulty, emotional role difficulty, energy/stamina/vitality, mental health, social functioning, and general health. When DQOL parameters were assessed, no statistically significant improvement was observed in satisfaction from training, psychological effects, social professional concerns, and diabetes-related concerns. Also, DTSQ parameters, such as satisfaction from treatment and frequency of hypo-/hyperglycemia, were similar before and after retraining.

\section{Discussion}

This study showed that user retraining increased the level of knowledge and application skills of patients with type 1 diabetes who were on CSII treatment. Insulin pump user retraining also improved glycemic parameters of patients with poor metabolic control.

Intensification of insulin treatment has been shown to be effective in the treatment of hyperglycemia in patients with type 1 diabetes [1-3]. As far as CSII users are concerned, further improvements could be achieved by user retraining [16]. Successful implementation of CSII requires a motivated and conscious patient having knowledge of technical skills and capability of self-management [18]. Candidates for CSII treatment should complete a well-organized training protocol that intensively covers both insulin pump management and carbohydrate counting skills. However, periodic re-evaluation and user retraining programs are essential for achieving a better glycemic control without increasing the risk of hypoglycemia. The use of self-assessment questionnaires may also allow patients to better appreciate their shortcomings on insulin pump management abilities [19].

The impact of chronic diseases as well as their treatment strategies on patients' QoL has been investigated extensively. Insulin pump therapy has been shown to improve general health, QoL, and treatment satisfaction of patients with type 1 diabetes $[6,9,20,21]$. QoL, functional health status, and satisfaction from treatment are thought to be affected by disease awareness and education of the individual patient. In our study, user retraining was associated with nonsignificant improvements in QoL and treatment satisfaction. However, we would presume that a better glycemic control will improve QoL in the long term as a result of potentially being protected from the complications of diabetes.

CSII User Retraining in Type 1 Diabetes
The limitations of this study include small sample size and a short follow-up period. Moreover, the training program was not personalized. Hence, further long-term studies including an individualized approach in a larger sample size are needed to determine the importance of retraining and planning retraining sessions at the beginning of pump usage.

Also, further studies considering patients with poorer metabolic control, patients with macrovascular complications, as well as patients of different ages should be conducted in order to observe the effects of retraining in these group of patients.

This study shows that the management of type 1 diabetes in insulin pump users can be significantly improved by retraining. Even a basic short-term retraining program helps patients increase their knowledge level and ability to more effectively use the insulin pump. We suggest that all patients with type 1 diabetes using CSII should be encouraged to take periodic retraining courses. The fact that retraining significantly improves glycemic parameters in patients with poor metabolic control indicates that priority should be given to this group of patients. Furthermore, being in constant communication would help diabetics cope with changes due to growing up or getting older, and would give them further ability to handle and control the disease than when newly diagnosed. Additionally, this would give a chance to follow up on technological developments. Further studies concerning more individualized re-education via telehealth, digital health, or the Internet should be pursued in order to assess the impact of individualized retraining and reach broader populations.

\section{Acknowledgements}

We thank our patients for participating in the study. Author contribution are as follows: B.O.S. researched data and wrote the manuscript and F.Y. designed the study and researched data. These two authors had equal contribution to the manuscript. The training courses were provided by N.A., B.B., and S.Y.; U.C. and S.O. researched data. B.A. designed the study, and reviewed and edited the manuscript. 


\section{References}

1 Diabetes Control and Complications Trial Research Group, Nathan DM, Genuth S, et al: The effect of intensive treatment of diabetes on the development and progression of longterm complications in insulin-dependent diabetes mellitus. The Diabetes Control and Complications Trial Research Group. N Engl J Med 1993;329:977-986.

2 Diabetes Control and Complications Trial/ Epidemiology of Diabetes Interventions and Complications Research Group, Lachin JM, et al: Retinopathy and nephropathy in patients with type 1 diabetes four years after a trial of intensive therapy. The Diabetes Control and Complications Trial/Epidemiology of Diabetes Interventions and Complications Research Group. N Engl J Med 2000;342:381389.

3 Nathan DM, Cleary PA, Backlund JY, et al: Intensive diabetes treatment and cardiovascular disease in patients with type 1 diabetes. N Engl J Med 2005;353:2643-2653.

4 Linkeschova R, Raoul M, Bott U, et al: Less severe hypoglycaemia, better metabolic control, and improved quality of life in type $1 \mathrm{di}-$ abetes mellitus with continuous subcutaneous insulin infusion (CSII) therapy; an observational study of 100 consecutive patients followed for a mean of 2 years. Diabet Med 2002;19:746-751.

5 Churchill JN, Ruppe RL, Smaldone A: Use of continuous insulin infusion pumps in young children with type 1 diabetes: a systematic review. J Pediatr Health Care 2009;23:173-179.
6 Thabit H, Hovorka R: Continuous subcutaneous insulin infusion therapy and multiple daily insulin injections in type 1 diabetes mellitus: a comparative overview and future horizons. Expert Opin Drug Deliv 2016;13: 389-400.

7 Misso ML, Egberts KJ, Page M, et al: Continuous subcutaneous insulin infusion (CSII) versus multiple insulin injections for type 1 diabetes mellitus. Cochrane Database Syst Rev 2010;3:CD005103.

8 Joshi M, Choudhary P: Multiple daily injections or insulin pump therapy: choosing the best option for your patient - an evidencebased approach. Curr Diab Rep 2015;15:81.

9 Abdul-Rasoul M, AlOtaibi F, Abdulla A, et al: Quality of life of children and adolescents with type 1 diabetes in Kuwait. Med Princ Pract 2013;22:379-384.

10 Clement S: Diabetes self-management education. Diabetes Care 1995;18:1204-1214.

11 Mitchell K, Johnson K, Cullen K, et al: Parental mastery of continuous subcutaneous insulin infusion skills and glycemic control in youth with type 1 diabetes. Diabetes Technol Ther 2013;15:591-595.

12 Grunberger G, Abelseth JM, Bailey TS, et al: Consensus Statement by the American Association of Clinical Endocrinologists/American College of Endocrinology insulin pump management task force. Endocr Pract 2014; 20:463-489.

13 American Association of Diabetes Educators: Continuous subcutaneous insulin infusion (CSII), AADE white paper. https://www.diabeteseducator.org/docs/default-source/legacydocs/_resources/pdf/publications/csii_pentulimate.pdf?sfvrsn=2 (issued June 6, 2014)
14 Parks L, Kim TY: Using remote communication technology in insulin pump training: a feasibility study. J Diabetes Sci Technol 2015; 10:398-404.

15 Samann A, Muhlhauser I, Bender R, et al: Glycaemic control and severe hypoglycaemia following training in flexible, intensive insulin therapy to enable dietary freedom in people with type 1 diabetes: a prospective implementation study. Diabetologia 2005;48:1965-1970.

16 Scheiner G, Sobel RJ, Smith DE, et al: Insulin pump therapy: guidelines for successful outcomes. Diabetes Educ 2009;35(suppl 2):29S41S; quiz 28S, 42S-43S.

17 Jenkins EJE, Knott J, Brooks A: Insulin pump users require recurrent education for the management of pump failure. J Diabetes Nurs 2016;20:364-369.

18 Aberle I, Scholz U, Bach-Kliegel B, et al: Psychological aspects in continuous subcutaneous insulin infusion: a retrospective study. J Psychol 2009;143:147-160.

19 Meade LT, Rushton WE: Optimizing insulin pump therapy: a quality improvement project. Diabetes Educ 2013;39:841-847.

20 Hoogma RP, Hammond PJ, Gomis R, et al: Comparison of the effects of continuous subcutaneous insulin infusion (CSII) and NPHbased multiple daily insulin injections (MDI) on glycaemic control and quality of life: results of the 5-nations trial. Diabet Med 2006; 23:141-147.

21 Rubin RR, Peyrot M, Group SS: Health-related quality of life and treatment satisfaction in the Sensor-Augmented Pump Therapy for A1C Reduction 3 (STAR 3) trial. Diabetes Technol Ther 2012;14:143-151. 